\title{
Imaging mass spectrometry enables molecular profiling of mouse and human pancreatic tissue
}

\author{
Boone M. Prentice ${ }^{1,2} \cdot$ Nathaniel J. Hart ${ }^{3} \cdot$ Neil Phillips $^{3} \cdot$ Rachana Haliyur $^{4} \cdot$ Audra Judd $^{2} \cdot$ Radhika Armandala $^{3}$. \\ Jeffrey M. Spraggins ${ }^{1,2,5} \cdot$ Cindy L. Lowe ${ }^{6} \cdot$ Kelli L. Boyd ${ }^{6} \cdot$ Roland W. Stein $^{4} \cdot$ Christopher V. Wright $^{7}$. \\ Jeremy L. Norris ${ }^{1,2,5} \cdot$ Alvin C. Powers $^{3,4,8} \cdot$ Marcela Brissova $^{3} \cdot$ Richard M. Caprioli $^{1,2,5,9}$
}

Received: 22 June 2018 / Accepted: 20 February 2019 / Published online: 6 April 2019

(C) Springer-Verlag GmbH Germany, part of Springer Nature 2019

\begin{abstract}
Aims/hypothesis The molecular response and function of pancreatic islet cells during metabolic stress is a complex process. The anatomical location and small size of pancreatic islets coupled with current methodological limitations have prevented the achievement of a complete, coherent picture of the role that lipids and proteins play in cellular processes under normal conditions and in diseased states. Herein, we describe the development of untargeted tissue imaging mass spectrometry (IMS) technologies for the study of in situ protein and, more specifically, lipid distributions in murine and human pancreases.

Methods We developed matrix-assisted laser desorption/ionisation (MALDI) IMS protocols to study metabolite, lipid and protein distributions in mouse (wild-type and $o b / o b$ mouse models) and human pancreases. IMS allows for the facile discrimination of chemically similar lipid and metabolite isoforms that cannot be distinguished using standard immunohistochemical techniques. Coregistration of MS images with immunofluorescence images acquired from serial tissue sections allowed accurate cross-registration of cell types. By acquiring immunofluorescence images first, this serial section approach guides targeted high spatial resolution IMS analyses (down to $15 \mu \mathrm{m}$ ) of regions of interest and leads to reduced time requirements for data acquisition.

Results MALDI IMS enabled the molecular identification of specific phospholipid and glycolipid isoforms in pancreatic islets with intra-islet spatial resolution. This technology shows that subtle differences in the chemical structure of phospholipids can dramatically affect their distribution patterns and, presumably, cellular function within the islet and exocrine compartments of the pancreas (e.g. 18:1 vs 18:2 fatty acyl groups in phosphatidylcholine lipids). We also observed the localisation of specific GM3 ganglioside lipids [GM3(d34:1), GM3(d36:1), GM3(d38:1) and GM3(d40:1)] within murine islet cells that were correlated with a higher level of GM3 synthase as verified by immunostaining. However, in human pancreas, GM3 gangliosides were equally distributed in both the endocrine and exocrine tissue, with only one GM3 isoform showing islet-specific localisation.

Conclusions/interpretation The development of more complete molecular profiles of pancreatic tissue will provide important insight into the molecular state of the pancreas during islet development, normal function, and diseased states. For example, this study demonstrates that these results can provide novel insight into the potential signalling mechanisms involving phospholipids and glycolipids that would be difficult to detect by targeted methods, and can help raise new hypotheses about the types of physiological
\end{abstract}

Electronic supplementary material The online version of this article (https://doi.org/10.1007/s00125-019-4855-8) contains peer-reviewed but unedited supplementary material, which is available to authorised users.

Richard M. Caprioli

richard.m.caprioli@vanderbilt.edu

19160 MRB III, Department of Biochemistry, Vanderbilt University, Nashville, TN 37232, USA

2 Mass Spectrometry Research Center, Vanderbilt University, Nashville, TN, USA

3 Division of Diabetes, Endocrinology and Metabolism, Vanderbilt University Medical Center, Nashville, TN, USA

4 Department of Molecular Physiology and Biophysics, Vanderbilt University, Nashville, TN, USA
5 Department of Chemistry, Vanderbilt University, Nashville, TN, USA

6 Translational Pathology Shared Resource, Vanderbilt University Medical Center, Nashville, TN, USA

7 Department of Cell \& Developmental Biology, Vanderbilt University, Nashville, TN, USA

8 Department of Veterans Affairs, Tennessee Valley Healthcare System, Nashville, TN, USA

9 Department of Pharmacology and Medicine, Vanderbilt University, Nashville, TN, USA 


\section{Research in context}

\section{What is already known about this subject?}

- Standard immunocytochemical techniques cannot alone access the broad array of molecular proteins, lipids and metabolites present in pancreatic tissue

- Lipids may play important roles in many aspects of islet dysfunction in diabetes, although in situ measurements of specific lipid isoforms are currently challenging owing to current methodological limitations

\section{What is the key question?}

- What is the distribution of specific lipids within pancreatic tissue and can imaging mass spectrometry (IMS) add to the molecular understanding of islet biology?

\section{What are the new findings?}

- High spatial resolution IMS enabled the localisation of specific protein and lipid isoforms to endocrine and exocrine tissue in both mouse and human pancreases

- Subtle differences in the chemical structure of phospholipids dramatically altered their spatial distribution in pancreatic tissue, probably resulting from different lipid biosynthesis pathways and/or the presence of different lipid isomers

- The presence of GM3 gangliosides in mouse islets and the differential expression of these lipids in human tissue further suggest that the role of gangliosides in insulin modulation may be species, organ and cell specific

How might this impact on clinical practice in the foreseeable future?

- These data demonstrate areas of islet biology where IMS can provide new and specific insight, moving beyond proteins to other classes of molecules, ultimately aiding in the design of novel strategies to sustain beta cell mass and function

control exerted on endocrine hormone-producing cells in islets. Importantly, the in situ measurements afforded by IMS do not require a priori knowledge of molecules of interest and are not susceptible to the limitations of immunohistochemistry, providing the opportunity for novel biomarker discovery. Notably, the presence of multiple GM3 isoforms in mouse islets and the differential localisation of lipids in human tissue underscore the important role these molecules play in regulating insulin modulation and suggest species, organ, and cell specificity. This approach demonstrates the importance of both high spatial resolution and high molecular specificity to accurately survey the molecular composition of complex, multi-functional tissues such as the pancreas.

Keywords Diabetes · Ganglioside $\cdot$ Imaging mass spectrometry $\cdot$ MALDI $\cdot$ Pancreas $\cdot$ Phospholipid

$\begin{array}{ll}\text { Abbreviations } \\ \text { FT-ICR } & \begin{array}{l}\text { Fourier transform } \\ \text { ion cyclotron resonance }\end{array} \\ \text { GM3 } & \begin{array}{l}\text { Monosialodihexosylganglioside } \\ \text { (NANA-Gal-Glc-ceramide) }\end{array} \\ \text { IHC } & \text { Immunohistochemistry } \\ \text { IMS } & \text { Imaging mass spectrometry } \\ \text { MALDI } & \text { Matrix-assisted laser desorption/ionisation } \\ \text { MS/MS } & \text { Tandem mass spectrometry } \\ m / z & \text { Mass-to-charge ratio } \\ \text { nanoDESI } & \text { Nanospray desorption electrospray ionisation } \\ \text { PC } & \text { Phosphatidylcholine } \\ \text { PE } & \text { Phosphatidylethanolamine } \\ \text { PI } & \text { Phosphatidylinositol } \\ \text { TIC } & \text { Total ion current }\end{array}$

\section{Introduction}

The ability of insulin-producing pancreatic beta $(\beta)$ cells to regulate blood glucose homeostasis is compromised in type 1 and type 2 diabetes [1, 2]. Despite substantial progress towards treatments and cures, much remains unknown about the molecular signatures of beta cell dysfunction [3, 4]. The anatomical location and small size of pancreatic islets, coupled with current methodological limitations, have prevented the development of a complete, coherent picture of the lipid and protein molecules that may be important in beta cell function and failure. These profiles would, for example, provide valuable information on the molecular signalling mechanisms affecting islet development throughout pancreas organogenesis and in a diseased state. 
Lipids are an important class of molecules that have long been implicated in many aspects of cellular dysfunction in diabetes, including mechanisms of apoptosis, beta cell cytokine secretion, impaired endoplasmic reticulum-to-Golgi vesicular trafficking, islet autoimmunity, and decreased insulin gene expression [5-9]. For example, ceramide and saturated fatty acids have both been demonstrated to induce beta cell lipo-apoptosis, though the specific ceramide lipids involved and the precise mechanisms of action remain unclear [7]. Phospholipids and glycolipids also play important roles in islet function. For example, the number of double bonds in the fatty acid tails of glycerophospholipids in the plasma membrane has been found to affect the function of glucose transport proteins [10-12]. Gangliosides, a class of glycosphingolipids characterised by one or more sialic acid residues, have been reported to participate in cellular proliferation, differentiation, and signal transduction and may serve as target antigenic molecules in type 1 diabetes [13, 14].

Unravelling the molecular signatures that define normal islet physiology and pathophysiology requires knowledge of both the identity and spatial localisation of biomolecules within the pancreas. Classical antibody-based immunohistochemistry (IHC) has a long and successful history in the analyses of biological tissue specimens due to the superb resolution and sensitivity of this technology. However, standard histological techniques alone cannot resolve the broad array of molecular compounds present in complex tissues, as many molecules are unknown or suitable antibodies cannot or have not been developed. The specific identity of lipids and metabolites is largely absent from such analyses because classical histological techniques usually do not resolve the isoforms of these molecules from one another. Additionally, approaches such as IHC only permit the detection of one molecule at a time (i.e. one molecule per antibody) and require a priori knowledge of the targeted molecule of interest, limiting their utility in the broad-based discovery of novel biomarkers or therapeutic target molecules in tissue. Even the most specific antibodies struggle to differentiate between highly similar molecules (e.g. lipid molecules that differ by only one double bond or by a few carbon atoms in fatty acid chain length).

One technology that offers a high degree of molecular specificity is mass spectrometry (MS). Previous MS approaches used to study the pancreas have employed tissue homogenisation and/or cellular fractionation prior to analysis, limiting the spatial information that can be obtained from such experiments [15-20]. Matrix-assisted laser desorption/ ionisation (MALDI) imaging mass spectrometry (IMS) utilises MS as an imaging modality and allows for the direct in situ molecular mapping of tissues without specialised reagents (i.e. antibodies) or any prior knowledge of the analytes present (Fig. 1b) [21]. In a MALDI IMS experiment, a chemical matrix with strong photon absorbance at the wavelength of the MALDI laser is applied to a thinly sectioned tissue specimen that is mounted onto a microscope slide. A raster of the sample surface is then performed to generate mass spectra at discrete $\mathrm{x}, \mathrm{y}$ positions across the tissue. Following data acquisition, maps of intensity can be reconstructed for any ion of interest [22-26]. Precise molecular identities are then confirmed by approaches such as accurate mass measurements and tandem mass spectrometry (MS/MS).

MALDI IMS has been used previously to successfully produce images of common pancreatic peptide hormones [21, 27-33]. However, IMS examinations of pancreatic lipid distributions have largely been restricted to studies of pancreatic cancer [34, 35]. Recently, nanospray desorption electrospray ionisation (nanoDESI) IMS has been used to study lipid distributions in a paraformaldehyde-fixed pancreas from a NOR/ LtJ mouse [36]. This technology utilises a continuous stream of solvent from a small capillary probe to entrain analyte molecules to the inlet of the mass spectrometer as the tissue surface is scanned with the probe and can distinguish between molecular signals from islets and the surrounding stroma and exocrine tissue [36]. Herein, we report the first application of MALDI IMS to the study of phospholipid and glycolipid distributions in pancreatic islets.

\section{Methods}

For detailed Methods, please refer to the electronic supplementary material (ESM) Methods.

Tissue handling Adult wild-type (wt/wt) and $o b / o b$ C57BL/6J mice were purchased from The Jackson Laboratory (Bar Harbor, ME, USA). At 8 weeks of age, fed and fasting blood glucose levels were measured from whole blood, taken from the tail, using an Accu-Chek glucose monitor (Roche Diagnostics, Indianapolis, IN, USA) (ESM Fig. 1). Mice were also weighed. The mice were euthanised and the pancreas was excised, weighed, and flash frozen by placing in aluminium foil prior to freezing in liquid nitrogen. All animal studies were reviewed and approved by the Institutional Animal Care and Use Committee at the Vanderbilt University Medical Center (see ESM Methods, Animals for further details).

The pancreas from non-cadaveric human donors aged 19 months (female donor) and 20 years (male donor) were obtained from the National Disease Research Interchange (NDRI) or the International Institute for the Advancement of Medicine (IIAM), respectively, as previously described (see ESM Methods, Human Samples for further details) [37, 38]. The Vanderbilt University Institutional Review Board declared that studies on de-identified human pancreatic specimens do not qualify as human subject research. All organs were stored at $-80^{\circ} \mathrm{C}$ until use. 


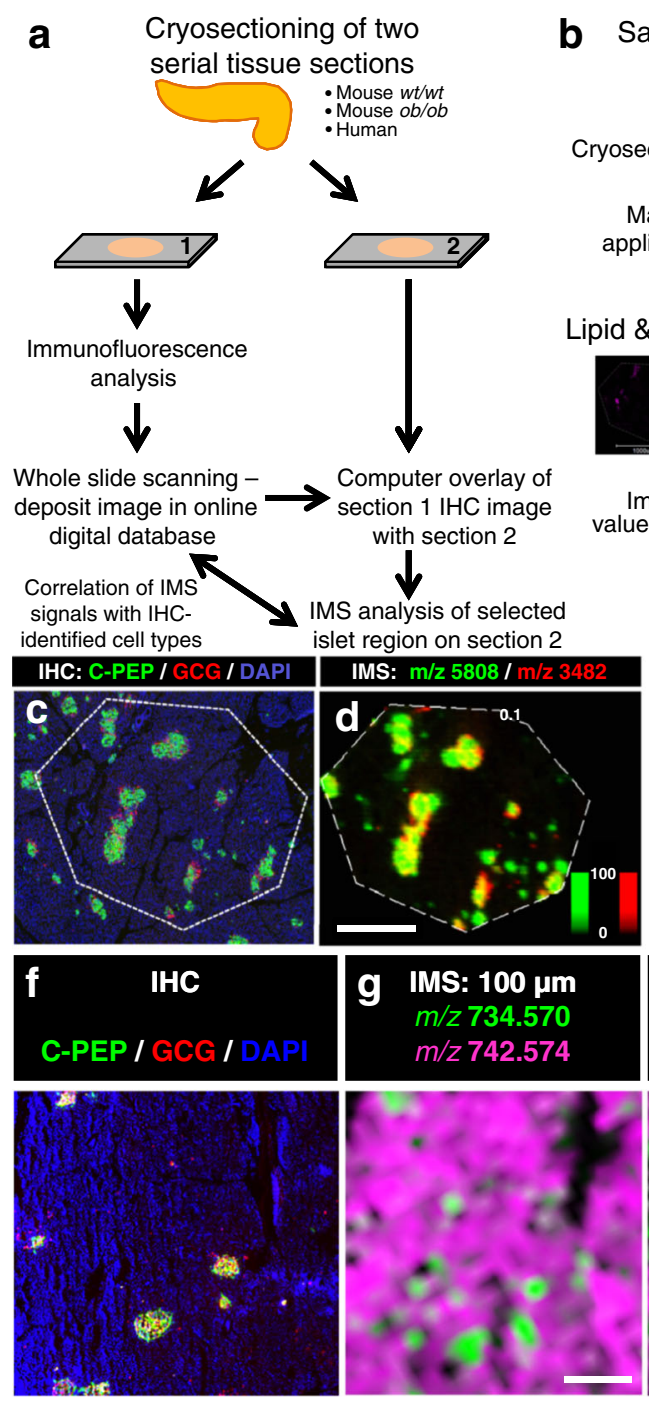

Fig. 1 Co-registration of IMS with fluorescence microscopy. (a) Serial tissue sections for immunofluorescence and IMS are prepared and coregistered to identify cell types and regions of interest. (b) The MALDI IMS workflow consists of first applying a chemical matrix homogenously across the sample. A raster of the surface performed with the MALDI laser provides for a mass spectrum at each $x, y$ position across the sample surface. Following data acquisition, heat maps of molecular intensity across the tissue are reconstructed for any ion of interest. Molecular identification is then confirmed using one or several MS-based technologies. (c, d) Co-registration of immunofluorescence (c) (green, C-peptide; red, glucagon; blue, DAPI) and IMS data (d) from a 19-month-old human donor enabled the identification of islets in IMS data. The false-colour IMS data acquired at $15 \mu \mathrm{m}$ spatial resolution validated the distribution of insulin (green, $m / z$ 5808) and glucagon (red, $m / z$ 3482). Ion images are displayed as $\pm 5 \mathrm{Da}$; an intensity scale of the false-colour IMS image is shown in the bottom right. (e) The red and green shading in the MS spectrum corresponds to the protein ions displayed in (d). In addition to

Tissue preparation and imaging analyses Frozen pancreases were sectioned at $10 \mu \mathrm{m}$ (unless otherwise noted) on a CryoStar NX70 Cryostat (Thermo Scientific, Waltham, MA, USA) at a chuck temperature of $-20^{\circ} \mathrm{C}$ and blade temperature of $-24^{\circ} \mathrm{C}$. Serial sections were alternatively thaw-mounted onto Superfrost Plus Gold Slides (Thermo Scientific) for
MALDI

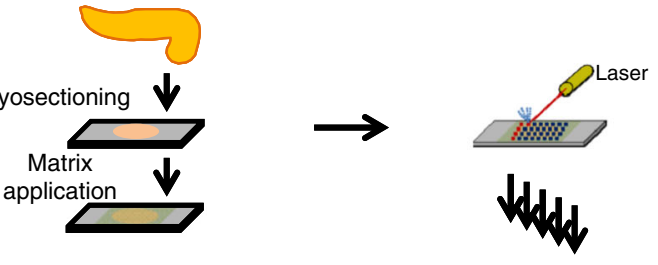

Data acquisition

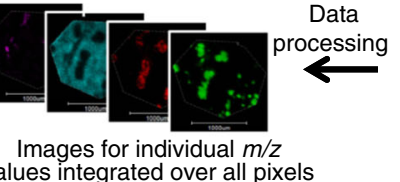

Analyte

-FT-ICR exact mass

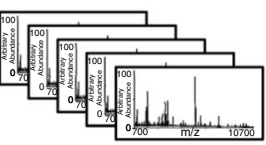

MS spectrum for each $x, y$

coordinate (pixel)
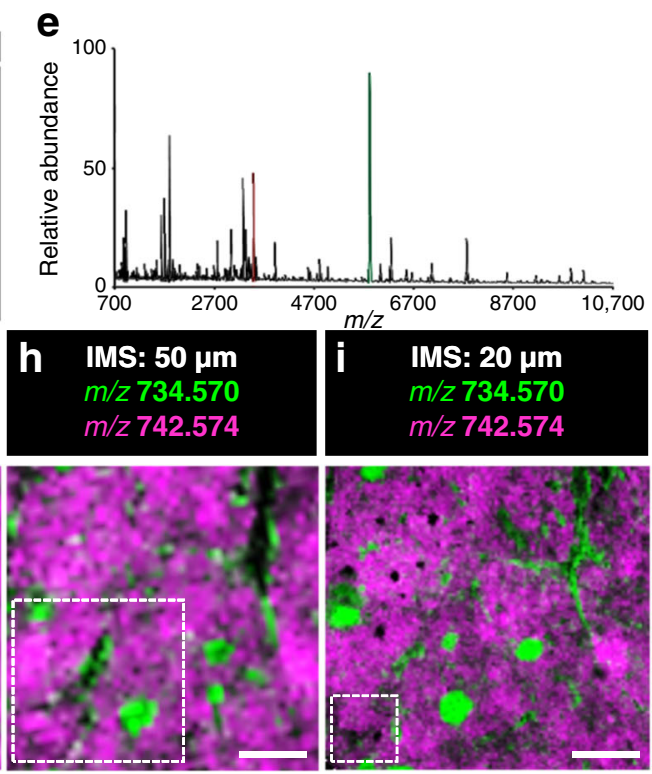

insulin and glucagon, protein IMS resulted in the detection of numerous other protein signals. (f-i) The spatial resolution of an IMS experiment is important in determining the level of structural detail that can be resolved in the resulting ion images (lipid images are displayed as $\pm 0.005 \mathrm{Da}$ ). Note that the use of $\mathrm{m} / \mathrm{z}$ values here is meant to highlight how mass spectral data are acquired. $\mathrm{m} / z .734 .570$ is likely to be $\mathrm{PC}(30: 0)$ and $\mathrm{m} / \mathrm{z}$ 742.574 is an isotope of an unidentified lipid that was chosen because of the clarity with which these two lipids show opposing spatial localisation, making the concept of spatial resolution (the purpose of this figure) easier to illustrate. Spatial resolutions of at least $30 \mu \mathrm{m}$ were used/required to adequately resolve islets. This co-registration workflow uses the immunofluorescence images to target subregions of interest for IMS and greatly reduced the acquisition time. Dotted white boxes in (h) and (i) depict the area that can be imaged in the same time as (g). All scale bars are $500 \mu \mathrm{m}$. C-PEP, C-peptide; GCG, glucagon. Portions of (b) are adapted with permission from B. M. Prentice, R. M. Caprioli and the Journal of Postdoctoral Research [39]

IHC and onto indium oxide (ITO)-coated glass slides for IMS (Delta Technologies, Loveland, CO, USA) and stored at $-80^{\circ} \mathrm{C}$ until use. Fresh frozen pancreas tissue sections were analysed either via immunofluorescence microscopy (see ESM Methods, Fluorescence microscopy for further details) or via MALDI IMS (see ESM Methods, Imaging Mass 
Spectrometry for further details). Briefly, fluorescence microscopy utilised rat $\alpha$-C-peptide, mouse $\alpha$-glucagon, goat $\alpha$ somatostatin primary antibodies for human sections and rat $\alpha$ C-peptide, rabbit $\alpha$-glucagon, goat $\alpha$-somatostatin primary antibodies for murine sections. Lipid IMS was performed on both human and murine tissue and protein IMS was performed on human tissue. GM3 synthase, insulin and glucagon immunochemistry was performed on flash-frozen fixed mouse pancreases to validate the GM3 lipid (a monosialodihexosylganglioside of the structure NANA-Gal-Glc-ceramide) observations (see ESM Methods, GM3 Synthase Immunochemistry for further details).

Statistical analysis IMS data are expressed as mean \pm SEM. Statistical analyses were performed using unpaired Student's $t$ test followed by post hoc Tukey test. Phosphatidylcholine quantification was performed by exporting average mass spectra from selected islet and exocrine regions of interest. $\mathrm{PC}$ ion intensities were normalised to a lipid ion expressed ubiquitously in endocrine and exocrine tissue, $\mathrm{PC}(36: 2)$. Ganglioside quantification was performed using ImageJ software (https:/imagej.nih.gov/ij/, National Institutes of Health, Bethesda, MD). Standard deviations (SDs) have also been calculated to assess reproducibility. Monochromatic ion images were normalised to total ion current (TIC) in FlexImaging and then exported to ImageJ, where signal intensity was measured as the mean intensity per islet of interest. The mean intensity and SEM were calculated for each ion of interest. Differences were considered statistically significant if $p<0.05$.

\section{Results}

IMS enables broad molecular coverage IMS analyses of the pancreas required several methodological considerations. First, to co-register the molecular signals detected in IMS experiments, we used a serial sectioning approach (Fig. 1a, b) in which one section of human donor tissue was analysed by fluorescence microscopy (Fig. 1c) and the adjacent section was analysed by high spatial resolution $(15 \mu \mathrm{m})$ IMS (Fig. 1d). This approach allowed us to confirm that the insulin and glucagon IMS signals were derived from beta cells and alpha cells, respectively (also shown in Fig. 2a, c, d). In addition to verifying islet location, accurate co-registration of immunofluorescent images enabled the acquisition of IMS data from selected subregions of the tissue, rather than of the entire tissue section. This spatially targeted acquisition is important for high spatial resolution IMS analyses because data acquisition time is inversely proportional to the square of the spatial resolution (i.e. larger imaged areas require longer acquisition times) [39]. This challenge is exacerbated on powerful high-resolution accurate mass instruments (e.g. the Fourier transform-ion cyclotron resonance [FT-ICR] mass spectrometer used herein) that are inherently slower than mass spectrometers capable of lower spectral performance (e.g. time-of-flight [TOF] mass spectrometers). Given that the spatial resolution required to adequately resolve islets is at least $\sim 30 \mu \mathrm{m}$ (Fig. 1f-i), the data acquisition times required for high spatial resolution IMS analyses of large tissue areas were impractical. For example, the amount of tissue that can be sampled at $50 \mu \mathrm{m}$ and $20 \mu \mathrm{m}$ spatial resolution in the same experimental time as the $100 \mu \mathrm{m}$ spatial resolution image (Fig. 1g) is represented by the white dotted lines in Fig. $1 \mathrm{~h}$ and i, respectively.

The untargeted nature of IMS technology enables simultaneous and much more complete measurement of the broad array of molecules present in a given cell population. Protein IMS analysis of human pancreas resulted in the unbiased detection of numerous protein signals directly from the tissue (Fig. 1e) and can be used to detect intact proteins up to $\sim 70 \mathrm{kDa}$. In addition to common endocrine hormones, many other proteins yet to be identified are also detected that are localised to other tissue structures including exocrine tissue and ductal tissue (Fig. 2b, e, f). Though the IMS spatial resolution used here $(15 \mu \mathrm{m})$ prevents precise single alpha and beta cell identification, magnifications of these images highlight the high-level co-registration between IMS and immunofluorescence data (magnified regions shown in Fig. 2a-f).

A unique capability of IMS is the facile discrimination of molecules with very similar chemical structures. For example, small differences in the double-bond content and length of the fatty acyl chains of glycerophospholipids can result in entirely different spatial distributions within the tissue (Fig. 2g-r). Positive-ion-mode lipid IMS analysis of a human pancreas resulted in the detection of many lipid signals, a small subset of which are shown in Fig. $2 \mathrm{~g}-1$. The phosphatidylcholines (PCs) PC(16:0_18:2) (Fig. 2h) and PC(16:0_18:1) (Fig. 2i) differ by only one double bond in one of the fatty acyl groups, yet these two lipids showed completely disparate localisation patterns, with the more saturated lipid, $\mathrm{PC}\left(16: 0 \_18: 1\right)$, localised to the islets and the more unsaturated lipid, $\overline{\mathrm{PC}}\left(16: 0 \_18: 2\right)$, localised to the surrounding exocrine tissue. Though these lipids are very similar in chemical structure and only differ by approximately $2 \mathrm{Da}$ in molecular mass, they are easily differentiated by FTICR IMS. Similarly, lipids that differ only in the total number of carbon atoms in one of the fatty acyl chains, such as PC(16:0_18:2) (Fig. 2h) and PC(18:0_18:2) (Fig. 2k), showed differential localisation within the tissue. Negative-ion-mode lipid IMS typically results in the detection of more acidic lipids, such as sulfatides and phosphatidylinositols (PIs) (Fig. 2m-r).

The ion images displayed in Fig. 2 are not meant to represent an exhaustive analysis and do not constitute a report of all lipids analysed; rather, these lipids merely exemplify the many instances of differential distribution that exist for a number of lipid classes (e.g. PCs, PIs, sulfatides) in pancreatic tissue. Analogous differences (i.e. differential lipid distributions within lipid classes) exist in mouse pancreases (see ESM 
Fig. 2 IMS enabled the untargeted detection of a wide array of molecular compounds in pancreatic tissue sections from a 19-month-old human donor. (a-f) Immunostaining (green, Cpeptide; red, glucagon; blue, DAPI) and false-colour $15 \mu \mathrm{m}$ spatial resolution IMS from serial tissue sections showing the spatial distribution of a series of proteins. Note that identification of the unknown proteins shown in (b, e, f) would require subsequent LCMS/MS experiments. These masses were simply selected to highlight the spatial molecular diversity detectable using MALDI IMS technology. (g-l) Immunostaining and false-colour $20 \mu \mathrm{m}$ spatial resolution IMS from serial tissue sections showing the spatial distribution of a series of PC ions. (m-r) Immunostaining and false-colour $20 \mu \mathrm{m}$ spatial resolution IMS from serial tissue sections showing the spatial distribution of a series of sulfatide and PI ions. Lipids were identified by a combination of accurate mass measurements (ppm error reported) and MS/MS (ESM Fig. 2). Ion images are shown without normalisation and with pixel interpolation as $\pm 0.005 \mathrm{Da}$. Magnifications of the areas highlighted with white dotted lines in the IHC images are shown below each IHC and IMS image. Intensity scales of the false-colour IMS images are shown in the bottom right of each magnified image. All scale bars are $100 \mu \mathrm{m}$. C-PEP, C-peptide; GCG, glucagon; SHexCer, sulfatide (sulfo-hexosyl-ceramide)

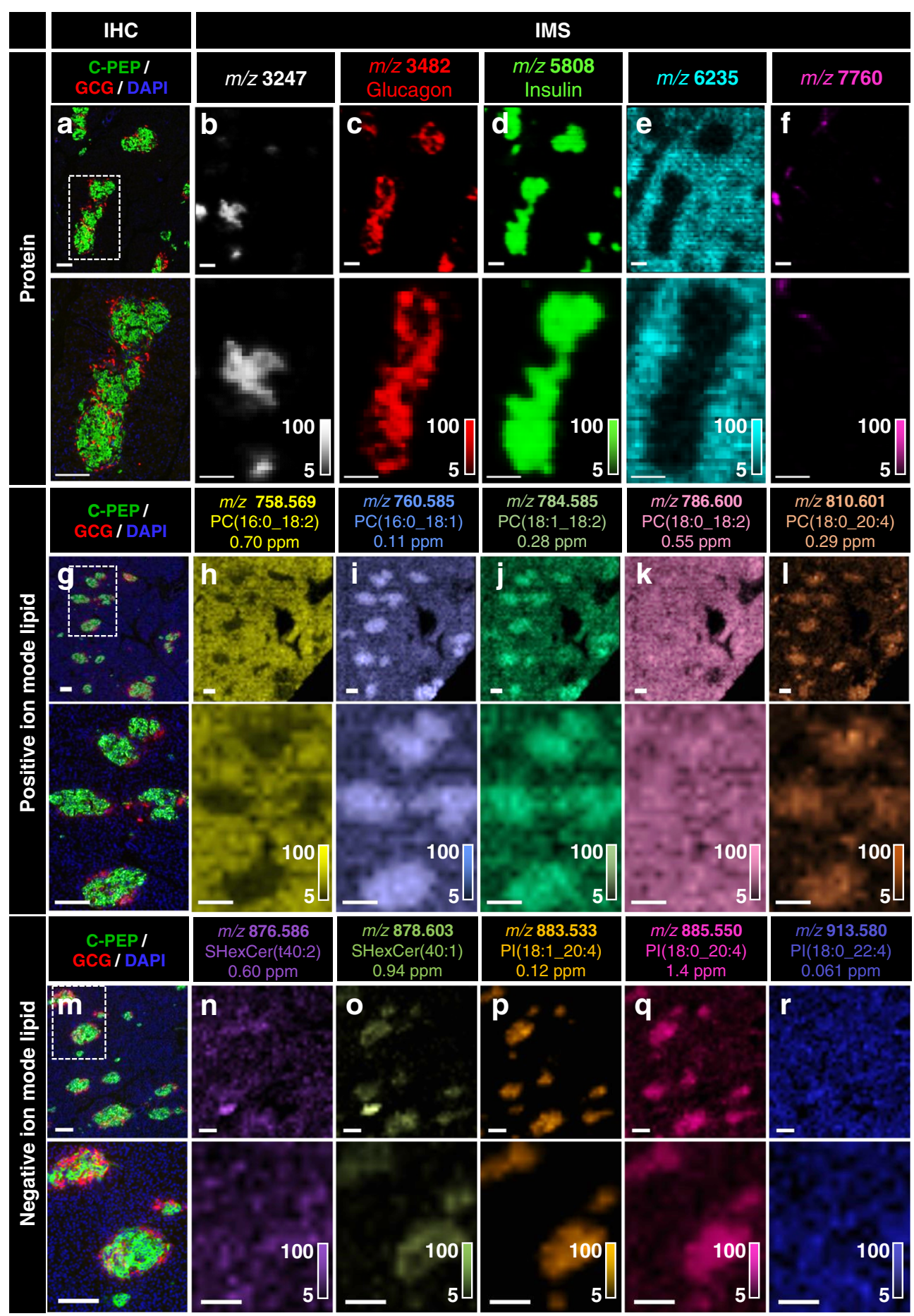

Table 1 for a list of some lipids detected in the single human pancreas donor studied here and ESM Table 2 for a list of some lipids detected in mouse pancreas; note that these are not exhaustive lists and the presence/absence of individual lipids in these two lists are not directly comparable as they were acquired under different sample preparation conditions). Interestingly, there are also differential distributions between the mouse and human tissues for the same mass-to-charge ratio $(\mathrm{m} / \mathrm{z})$ compound (data not shown). However, an exhaustive comparison of mouse and human pancreas lipid distributions is beyond the scope of this initial report. These differences are likely the result of different lipid biosynthesis pathways and/or different lipid isomers and highlight the importance of molecular specificity in these types of imaging measurements. An imaging modality that could not differentiate between these chemical species would produce images representing the confluence of multiple lipids and would preclude the acquisition of this finer molecular detail. 
IMS reproducibility To assess IMS reproducibility, positiveion-mode PC lipid distributions were analysed under conditions of controlled biological variance (ESM Fig. 1). Pancreases from three $w t / w t$ mice and three $o b / o b$ mice were each analysed by IMS (Fig. 3). Similar to human pancreatic tissue, co-registration of PC lipid IMS data with an IHC image allowed for the identification of endocrine and exocrine regions (Fig. 3a-j). Normalisation of PC(34:2), PC(34:1) and $\mathrm{PC}(38: 4)$ signals to $\mathrm{PC}(36: 2)$, an ion expressed ubiquitously across endocrine and exocrine regions, allowed for relative quantification of lipids within islet and exocrine regions. Five regions of interest (islet and exocrine regions) from each biological replicate for both $w t / w t$ and $o b / o b$ tissue were measured (Fig. 3k). Upon normalisation, the relative SDs of the exocrine tissue regions for both the $w t / w t$ tissue $[\mathrm{PC}(34: 1)=5.2 \%, \mathrm{PC}(34: 1)=7.6 \%$, and $\mathrm{PC}(38: 4)=8.4 \%]$ and $o b / o b$ tissue $[\mathrm{PC}(34: 1)=9.6 \%, \mathrm{PC}(34: 1)=7.9 \%$, and $\mathrm{PC}(38: 4)=15.6 \%]$ were much smaller than the relative SDs of the islet tissue regions $[\mathrm{PC}(34: 1)=31.0 \%, \mathrm{PC}(34: 1)=$ $12.2 \%$, and $\mathrm{PC}(38: 4)=14.3 \%$ for $w t / w t$ and $\mathrm{PC}(34: 1)=$ $37.2 \%, \mathrm{PC}(34: 1)=39.2 \%$, and $\mathrm{PC}(38: 4)=32.0 \%$ for $o b / o b]$, highlighting the heterogeneity of the lipid composition between islets compared with exocrine tissue. The abundance of each of these three lipids differed significantly between the islet and exocrine tissue regions (Fig. 3k). There was no statistically significant difference in PC
Fig. 3 IMS was reproducible and allowed for relative quantification of lipids of interest. (a-e) Immunostaining (green, Cpeptide; red, glucagon; blue, DAPI) and false-colour $30 \mu \mathrm{m}$ spatial resolution IMS from serial tissue sections showing the spatial distribution of a series of PC ions in $w t / w t$ mouse pancreatic tissue. (f-j) Immunostaining (colours as in a) and false-colour $30 \mu \mathrm{m}$ spatial resolution IMS from serial tissue sections showing the spatial distribution of a series of PC ions in $o b / o b$ mouse pancreatic tissue. Lipids were identified by accurate mass measurements (ppm error reported). Ion images have been normalised to PC(36:2) (a lipid expressed homogenously throughout each tissue) and are shown with pixel interpolation as \pm 0.005 Da. Magnifications of the areas highlighted with white dotted lines in the IHC images are shown below each IHC and IMS image. Intensity scales of the false-colour IMS images are shown in the bottom right of each magnified image. (k) Intensity values (I), presented as mean \pm SEM, represent the average of five islets each from three biological replicates (15 total islets) in each tissue type (***p $\leq$ 0.001). PC signals were normalised to $\mathrm{PC}(36: 2)$. All scale bars are $100 \mu \mathrm{m}$. C-PEP, Cpeptide; GCG, glucagon
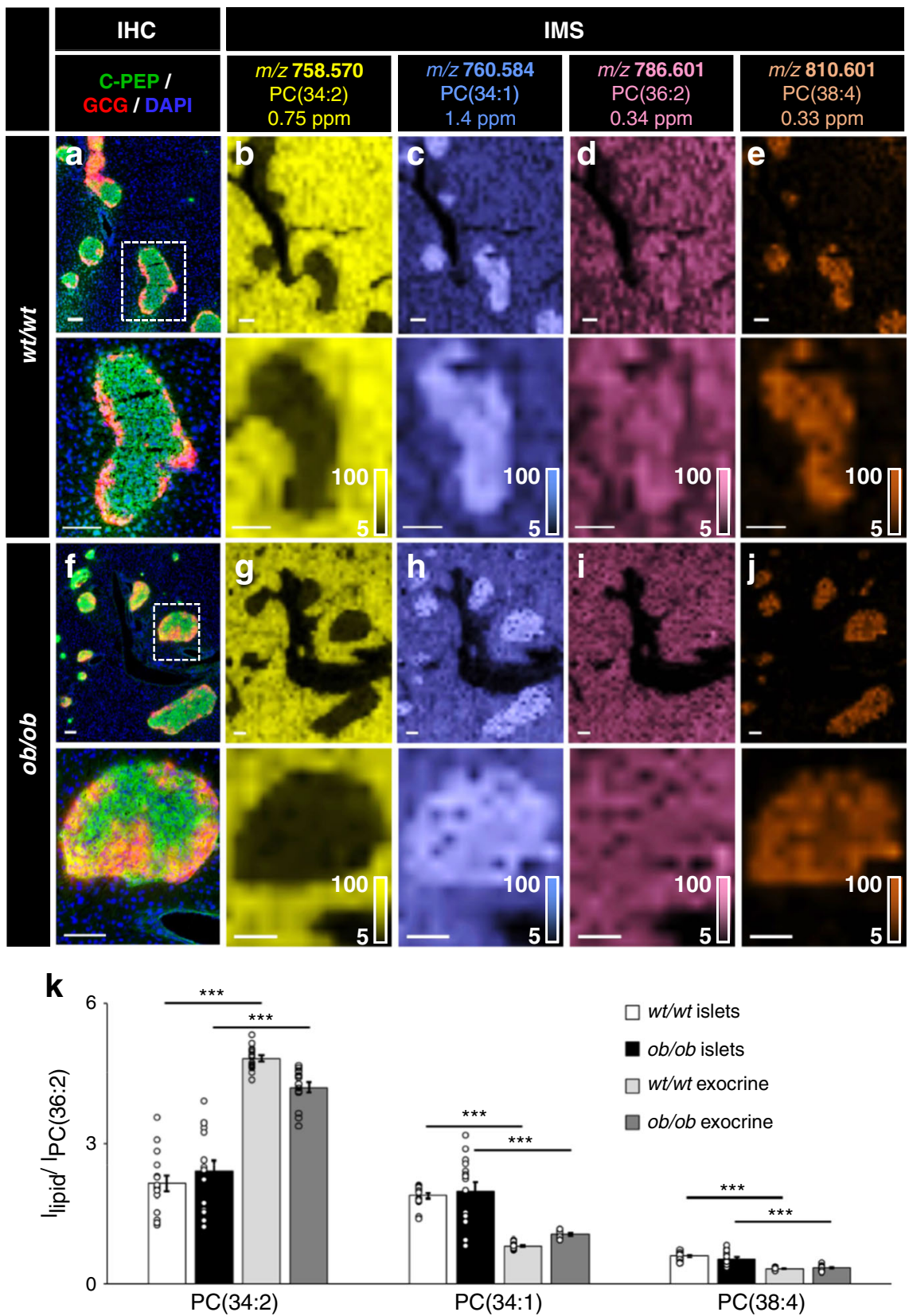
abundances between $w t / w t$ and $o b / o b$ islets, and only $\operatorname{PC}(34: 2)$ and $\mathrm{PC}(34: 1)$ were significantly different between $w t / w t$ and $o b / o b$ exocrine tissue (not indicated in Fig. 3k).

Ganglioside IMS In addition to differences between endocrine and exocrine expression levels, we also observed differential lipid distributions within islets. Though most lipid classes showed minimal intra-islet heterogeneity (e.g. PC, phosphatidylethanolamine [PE] and PI lipids noted above), high spatial resolution lipid IMS analysis showed that the ganglioside GM3(d34:1) was more abundant in the periphery of $w t / w t$ and $o b / o b$ mouse islets (see magnified images in Fig. $4 \mathrm{~b}, \mathrm{~g}$ ).
Co-registration of these lipid images with fluorescence microscopy suggested that the elevated lipid levels were associated with alpha cells (see magnified images in Fig. 4a, f). However, given that the IMS spatial resolution was $30 \mu \mathrm{m}$ and that the immunofluorescence and IMS images were acquired from serial tissue section, single-cell registration was not feasible. A number of other GM3 lipids were also detected in both $w t / w t$ and $o b / o b$ mouse islets, including GM3(d36:1), GM3(d38:1) and GM3(d40:1) (Fig. 4a-j). In both the $w t / w t$ and $o b / o b$ mouse tissue, all four GM3 gangliosides were localised to the islets, and likely enriched in the alpha cells because of their apparent localisation to the islet periphery. In order to
Fig. 4 IMS enabled the detection of specific GM3 ganglioside isoforms in murine pancreases. (a-e) Immunostaining (green, Cpeptide; red, glucagon; blue, DAPI) and false-colour $30 \mu \mathrm{m}$ spatial resolution IMS from serial tissue sections showing the spatial distribution of a series of GM3 ganglioside ions in $w t / w t$ mouse pancreatic tissue. $(\mathbf{f}-\mathbf{j})$ Immunostaining (colours as in a) and false-colour $30 \mu \mathrm{m}$ spatial resolution IMS from serial tissue sections showing the spatial distribution of a series of GM3 ganglioside ions in $o b / o b$ mouse pancreatic tissue. Lipids were identified by accurate mass measurements (ppm error reported). Ion images were normalised to TIC and are shown with pixel interpolation as \pm 0.05 Da. Magnifications of the areas highlighted with white dotted lines in the IHC images are shown below each IHC and IMS image. Intensity scales of the false-colour IMS images are shown in the bottom right of each magnified image. (k) Intensity values, presented as mean $\pm \mathrm{SEM}$, represent the average of ten islets each from five biological replicates (50 total islets) in each tissue type $(* * * p \leq 0.001)$. GM3 signals were normalised to TIC prior to data export. (1-o) GM3 synthase immunostaining of serial tissue sections of a $w t / w t$ mouse pancreas (l, insulin; m, GM3 synthase) and an $o b / o b$ mouse pancreas (n, insulin; o, GM3 synthase). All scale bars are $100 \mu \mathrm{m}$. C-PEP, C-peptide; GCG, glucagon
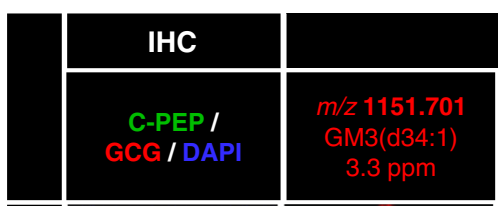

\begin{tabular}{|c|c|}
\hline \multicolumn{2}{|c|}{ IMS } \\
\hline m/z 1179.737 & m/z 1207.769 \\
GM3(d36:1) & GM3(d38:1) \\
$0.95 p p m$ & $1.5 p p m$ \\
\hline
\end{tabular}

$\mathrm{m} / \mathrm{z} 1235.800$ GM3(d40:1) $0.23 \mathrm{ppm}$
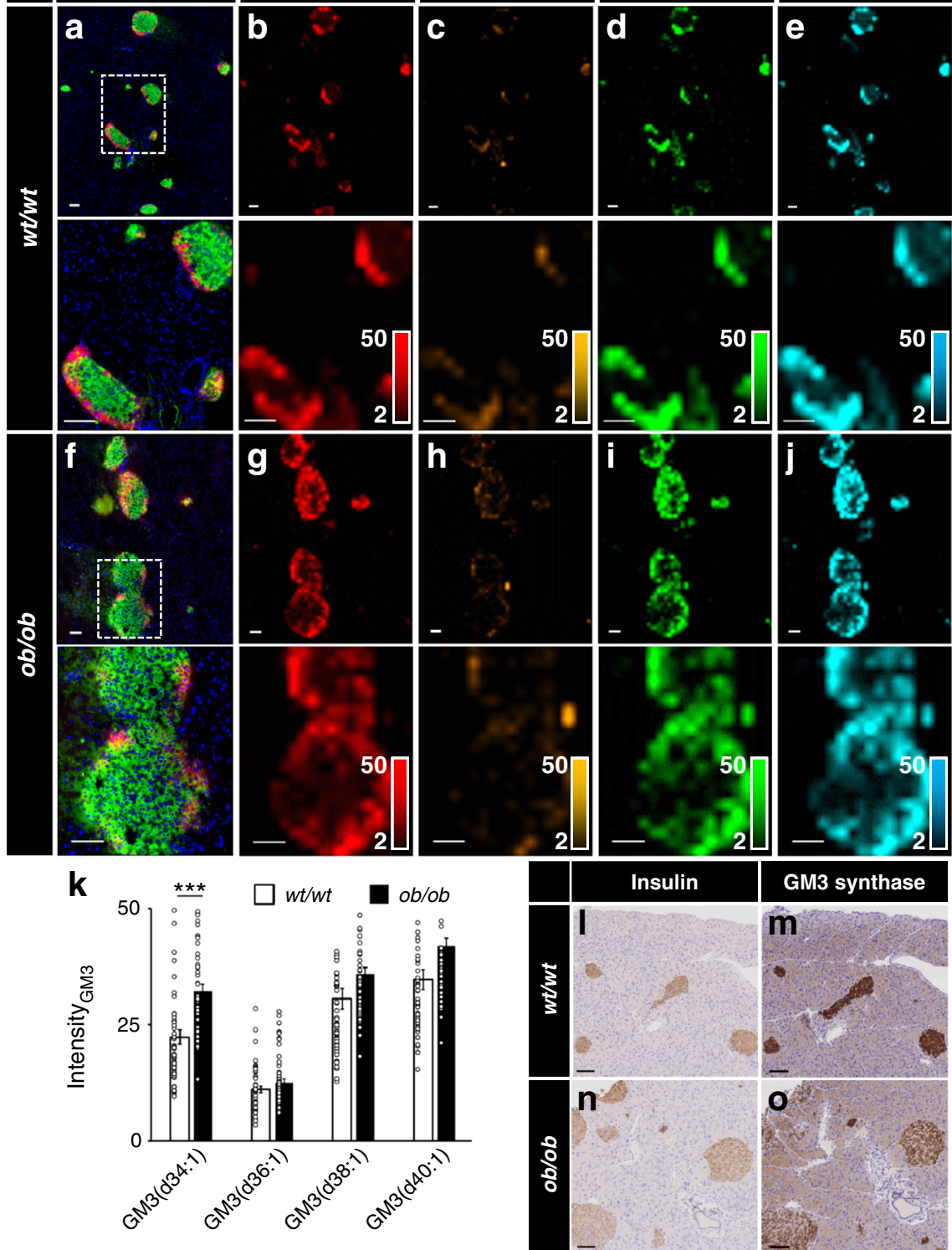
quantitatively compare the expression levels of these GM3 lipids in $w t / w t$ and $o b / o b$ mouse islets, ten islets each were selected from five biological replicates (50 total islets) for each tissue type. The signal for each GM3 lipid was normalised to the TIC, allowing for relative comparisons to be made between the samples. We detected some differences in the islet distributions of these gangliosides (Fig. 4k). However, as noted for PC quantification, the SDs for these islet measurements were quite large due to inter-islet heterogeneity. It should also be noted that the relative quantification approach used here allows for reliable lipid comparisons between tissue types. Absolute quantification of lipid levels in tissue by IMS would require the use of carefully selected internal standards, the use of proper calibration curves, and validation by an established method (e.g. LC-MS/MS), which is beyond the scope of the current report.

In order to validate our detection of islet-restricted gangliosides using IMS, we stained both $w t / w t$ and $o b / o b$ mouse pancreatic tissue for GM3 synthase, the enzyme responsible for GM3 biosynthesis (Fig. 41-0). This enzyme produces GM3 gangliosides by catalysing the addition of sialic acid onto lactosylceramide [14]. GM3 is the simplest and most abundant ganglioside, serving as a metabolic precursor for more complex a-, b- and c-series gangliosides, which can all degrade via the sialidase-mediated hydrolytic cleavage of sialic acid chains to again produce GM3 [14]. The apparent elevation of GM3 lipid abundances in alpha cells compared with beta cells could be due to differences in some of these synthesis and degradation pathways. Our results demonstrate that GM3 synthase expression was largely restricted to the islets of both tissues, suggesting that GM3 production and localisation occur almost exclusively in islets, the consequences of which are not yet known.

Interestingly, the spatial distributions of the GM3 gangliosides detected in the mouse pancreases were not conserved in human tissue (Fig. 5). Specifically, GM3(d36:1), GM3(d38:1) and GM3(d40:1) were ubiquitously distributed throughout the human pancreas (Fig. 5c-e). Only GM3(d34:1) was localised to the islets in both the mouse and human pancreas samples (Fig. 5b). Additionally, while GM3(d34:1) was localised to the periphery of the mouse islets, this lipid was expressed relatively evenly throughout the human islets (see magnified images in Fig. 5b), possibly reflecting that alpha cells are distributed throughout the human islet. The differences in spatial distribution of these ganglioside lipids in human tissue highlights the importance of molecular specificity in IMS.

\section{Discussion}

Molecular studies in targeted diagnostics and therapeutics have historically been limited to systems with some a priori knowledge of the specific molecules or biological pathways to be studied. However, new technologies emerging in recent years have allowed more systematic, comprehensive, and unbiased studies of biological and clinical samples, in turn enabling greater insights into basic cellular biology and offering new opportunities for curative therapies. In recent years, MALDI IMS has proven useful in areas of study such as cancer diagnosis and classification, drug distribution determination in tissue, and biomarker identification [24, 26]. As exemplified in this work, MALDI IMS can now be used to determine the molecular composition of islets in intact pancreatic tissue sections. We have shown that reproducible lipid measurements can be made across multiple biological replicate tissues using appropriate normalisation protocols, although the native biological variability of islets can make these molecular comparisons challenging. The rapid processing, flash-freezing, and fixing protocols used here during pancreas procurement and sample preparation were crucial to maintaining molecular fidelity in the tissue to enable the acquisition of high-quality IMS and immunofluorescence measurements. As the spatial resolution of IMS technology is improved further, individual islet cells and even cellular organelles could be targeted for interrogation. Though there are obviously limitations to any analytical technology (e.g. MALDI IMS requires the addition of a carefully chosen chemical matrix to ensure optimal sensitivity towards a desired class of analytes and MALDI IMS spatial resolution and sensitivity are currently inferior to fluorescence-based imaging modalities [spatial resolution of $20 \mu \mathrm{m}$ for IMS vs $<1 \mu \mathrm{m}$ for immunofluorescence]), the regioselective molecular specificity afforded by MALDI IMS offers a new data dimension in studies of health and disease. Herein, we have used novel MALDI IMS methodologies to identify the localisation of specific phospholipid and glycolipid species in pancreatic islets.

Roughly $75 \%$ of the plasma membrane is comprised of phospholipids, of which approximately 50\% are PCs [40]. Though these molecules are primarily structural lipids, MALDI IMS here reveals remarkable differences in endocrine and exocrine PC spatial localisation in pancreatic tissue depending on the total carbon length and the number of double bonds in the fatty acyl lipid groups (Figs $2 \mathrm{~h}-1$ and 3 ). Some of these lipids show similar spatial distributions to those reported in mouse pancreas by the Laskin group, using nanoDESI IMS [36]. Recent reports have indicated that increased lipid saturation in the plasma membrane can cause increased membrane rigidity, which in turn can disrupt vesicle fusion and glucose transport protein activity (GLUT) in type 2 diabetes [12]. The degree of unsaturation of NEFA is also known to affect the role of these lipids in beta cell apoptosis and proliferation [10, 11, 41]. For example, arachidonic acid-containing phospholipids have been suggested to serve as important substrates for beta cell phospholipases (Fig. 21, p, q) $[10,11]$. Though the phospholipid results reported here provide only a small 

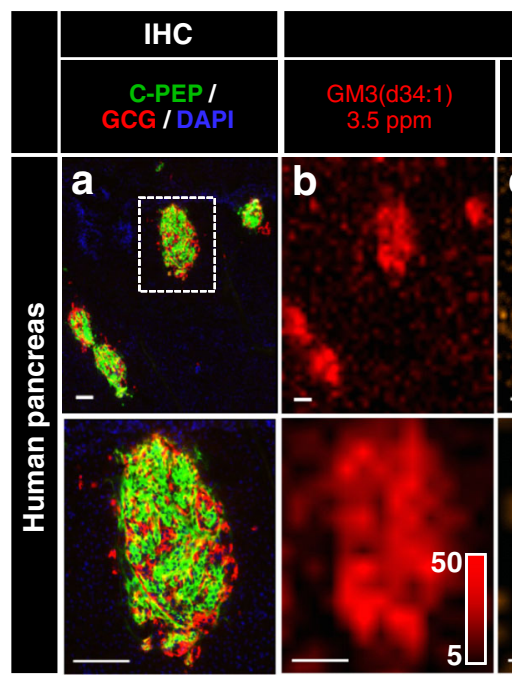

Fig. 5 IMS enabled the detection of specific GM3 ganglioside isoforms in a human pancreas. Immunostaining (green, C-peptide; red, glucagon; blue, DAPI) and false-colour $30 \mu \mathrm{m}$ spatial resolution IMS from serial tissue sections showing the spatial distribution of a series of GM3 ganglioside ions in human pancreatic tissue from a 20 -year-old donor. Lipids were identified by accurate mass measurements (ppm error reported). Ion

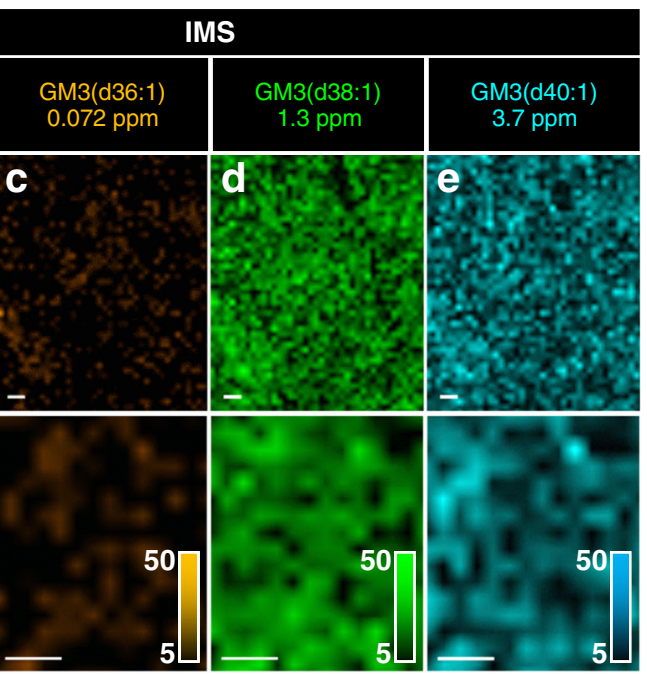

images were normalised to TIC and are shown with pixel interpolation as $\pm 0.05 \mathrm{Da}$. Magnifications of the area highlighted with white dotted lines in the IHC image are shown below each IHC and IMS image. Intensity scales of the false-colour IMS images are shown in the bottom right of each magnified image. All scale bars are $100 \mu \mathrm{m}$. C-PEP, C-peptide; GCG, glucagon

snapshot of lipid profiles in the pancreas, the dramatic differences in the localisation of these phospholipids highlight the importance of spatial resolution and molecular specificity in understanding the precise molecular signatures of islets and warrant future investigation.

Our investigation of pancreatic tissue revealed several differences in ganglioside distribution throughout the pancreas. This is of particular interest, as gangliosides are known modulators of transmembrane signalling and act as autoantigens in a number of autoimmune diseases, though literature reports of these roles vary depending on the organ and species under study. For example, GM2-1 has been suggested as an antigen of cytoplasmic islet cell autoantibodies (ICA) [42]. Interestingly, Dotta and co-workers demonstrated that GM2-1 is expressed in the secretory granules of rat islets and is not necessarily beta cell specific [43]. Additionally, there is growing evidence that gangliosides fulfil other important metabolic roles such as proliferation, differentiation, and signal transduction [14]. In particular, GM3 ganglioside accumulation and GM3 synthase activity have been detected in the adipose tissue of $o b / o b$ mice [13]. GM3 has also been shown to be abundant in the pancreatic exocrine tissue of rats, but curiously absent from the islets [44]. This and other studies suggest that the role of GM3 in insulin modulation may be species, organ, and/or celltype dependent [45-47]. Additionally, it is unclear which specific GM3 isoforms play a role in insulin secretion, the onset of obesity, and/or diabetes loss of function because the studies performed to date have used technologies such as thin-layer chromatography, immunostaining, and HPLC coupled to ultraviolet detection, which do not distinguish between different chemical structures within a class of molecules (e.g. GM3 gangliosides with different fatty acid chains). MALDI IMS can provide this high degree of molecular specificity in the form of an image produced in situ, circumventing the need to homogenise the entire tissue prior to analysis.

Here, MALDI IMS has shown that GM3 gangliosides in mouse tissue are specific to the pancreatic islets and are largely absent from the surrounding exocrine tissue, localisations which are not conserved in human tissue. Differences in cell organisation between species may contribute to the observed differences in lipid distributions [48, 49]. Moreover, those GM3 lipids that are localised to islets in murine pancreases appear to be elevated in alpha cells compared with beta cells. Gangliosides in the pancreas are thought to reside mainly in secretory granules [43]. These results could suggest that GM3 is elevated in glucagon secretory granules compared with insulin secretory granules. Additionally, the GM3 isoforms detected here contain only saturated fatty acid tails, which could act to limit the ability of saturated NEFA to induce beta cell apoptosis. Though the differences in expression between wild-type and $o b / o b$ mouse islets could imply a role for GM3 involvement in beta cell dysfunction, at least in this mouse model, the highly heterogeneous nature of these islet measurements precludes a definitive conclusion. The analysis of a limited number of human samples in this study precludes firm conclusions on IMS distributions in human samples and warrants further investigation. Regardless, the presence of GM3 in islet cells suggests that these gangliosides could be important in endocrine hormone signalling.

Conclusions MALDI IMS has enabled the identification of specific phospholipid and glycolipid isoforms present in 
pancreatic islets. These studies exemplify the importance of both spatial resolution and specificity when making molecular measurements of complex heterogeneous tissues. Whereas studies that homogenise the entire organ are unable to provide exocrine vs endocrine spatial resolution, MALDI IMS enables intra-islet resolution and shows that subtle changes in the chemical structure of phospholipids can dramatically affect the distribution and, presumably, cellular function of these lipids. The in situ measurements afforded by IMS do not require a priori knowledge of molecules of interest and are not susceptible to non-specific antibody binding, providing the opportunity to study many other novel protein, lipid and metabolite molecules across different stages of pancreas development and in various disease states.

Data availability All data are available from the authors upon reasonable request (data are retained for 5 years).

Funding This work was supported by the National Institutes of Health (NIH) under award P41 GM103391-07 (National Institute of General Medical Sciences [NIGMS]) and by the National Institute of Diabetes and Digestive and Kidney Diseases (NIDDK)-supported Human Islet Research Network (HIRN, RRID:SCR_014393; https:/hirnetwork.org; UC4DK104211, DK108120, DK104218 and DK112232), DK106755, DK72473, DK89572, DK97829, DK94199, the Vanderbilt Diabetes Research and Training Center (DK20593) and grants from the JDRF, The Leona M. and Harry B. Helmsley Charitable Trust, and the Department of Veterans Affairs. TPSR is supported by the NIH under awards 5P30 CA68485-19 (National Cancer Institute [NCI]) and 2U24 DK059637-16 (Vanderbilt Mouse Metabolic Phenotyping Center). BMP was supported by the NIH/NIDDK under award F32 FDK105841A.

Duality of interest The authors declare that there is no duality of interest associated with this manuscript.

Contribution statement BMP, NJH, NP, RH, MB, JLN, ACP and RMC conceived and designed the research. NJH and NP performed glucose tolerance tests and animal euthanasia. BMP and AJ prepared tissue sections. BMP performed MALDI IMS and MS/MS analyses. NJH, NP, RH, $\mathrm{AJ}$ and RA conceived experiments and performed hormone IHC. AJ, CLL and KLB performed GM3 synthase IHC. BMP analysed MS data. BMP, NJH, NP, RH, JMS, RWS, CVW, MB, JLN, ACP and RMC interpreted results of experiments. BMP prepared figures and drafted the manuscript prior to revision and approval by the other authors. $\mathrm{RMC}$ is the guarantor of this work.

\section{References}

1. Slack J (1995) Developmental biology of the pancreas. Development 121:1569-1580

2. Prado C, Pugh-Bernard A, Elghazi L, Sosa-Pineda B, Sussel L (2004) Ghrelin cells replace insulin-producing beta cells in two mouse models of pancreas development. Proc Natl Acad Sci U S A 101(9):2924-2929. https://doi.org/10.1073/pnas.0308604100

3. Wang Q, Jin T (2009) The role of insulin signaling in the development of $\beta$-cell dysfunction and diabetes. Islets 1(2):95-101. https:// doi.org/10.4161/isl.1.2.9263

4. Talchai C, Lin H, Kitamura T, Accili D (2009) Genetic and biochemical pathways of beta-cell failure in type 2 diabetes. Diabetes
Obes Metab 11(Suppl 4):38-45. https://doi.org/10.1111/j.14631326.2009.01115.x

5. Buschard K, Josefsen K, Hansen S et al (1994) Sulphatide in islets of Langerhans and in organs affected in diabetic late complications: a study in human and animal tissue. Diabetologia 37(10):1000 1006. https://doi.org/10.1007/BF00400463

6. Lang F, Ullrich S, Gulbins E (2011) Ceramide formation as a target in beta-cell survival and function. Expert Opin Ther Targets 15(9): 1061-1071. https://doi.org/10.1517/14728222.2011.588209

7. Boslem E, Meikle P, Biden T (2012) Roles of ceramide and sphingolipids in pancreatic $\beta$-cell function and dysfunction. Islets 4(3):177-187. https://doi.org/10.4161/isl.20102

8. Galadari S, Rahman A, Pallichankandy S, Galadari A, Thayyullathil F (2013) Role of ceramide in diabetes mellitus: evidence and mechanisms. Lipids Health Dis 12(1):98. https://doi.org/ 10.1186/1476-511X-12-98

9. Russo S, Ross J, Cowart L (2013) Sphingolipids in obesity, type 2 diabetes, and metabolic disease. Handb Exp Pharmacol 216:373-401

10. Hsu F, Bohrer A, Wohltmann M et al (2000) Electrospray ionization mass spectrometric analyses of changes in tissue phospholipid molecular species during the evolution of hyperlipidemia and hyperglycemia in Zucker diabetic fatty rats. Lipids 35(8):839-854. https://doi.org/10.1007/S11745-000-0593-Z

11. Ramanadham S, Hsu F, Zhang S, Bohrer A, Ma Z, Turk J (2000) Electrospray ionization mass spectrometric analyses of phospholipids from INS-1 insulinoma cells: comparison to pancreatic islets and effects of fatty acid supplementation on phospholipid composition and insulin secretion. Biochim Biophys Acta 1484(2-3):251266. https://doi.org/10.1016/S1388-1981(00)00022-6

12. Weijers RN (2012) Lipid composition of cell membranes and its relevance in type 2 diabetes mellitus. Curr Diabetes Rev 8(5):390400. https://doi.org/10.2174/157339912802083531

13. Tagami S, Inokuchi J, Kabayama K et al (2002) Ganglioside GM3 participates in the pathological conditions of insulin resistance. J Biol Chem 277(5):3085-3092. https://doi.org/10.1074/jbc.M103705200

14. Lipina C, Hundal HS (2015) Ganglioside GM3 as a gatekeeper of obesity-associated insulin resistance: evidence and mechanisms. FEBS Lett 589(21):3221-3227. https://doi.org/10.1016/j.febslet. 2015.09.018

15. Metz TO, Jacobs JM, Gritsenko MA et al (2006) Characterization of the human pancreatic islet proteome by two-dimensional LC/ MS/MS. J Proteome Res 5(12):3345-3354. https://doi.org/10. 1021/pr060322n

16. Petyuk VA, Qian WJ, Hinault $C$ et al (2008) Characterization of the mouse pancreatic islet proteome and comparative analysis with other mouse tissues. J Proteome Res 7(8):3114-3126. https://doi. org $/ 10.1021 / \mathrm{pr} 800205 \mathrm{~b}$

17. Waanders LF, Chwalek K, Monetti M, Kumar C, Lammert E, Mann M (2009) Quantitative proteomic analysis of single pancreatic islets. Proc Natl Acad Sci U S A 106(45):18902-18907. https://doi. org/10.1073/pnas.0908351106

18. El Ouaamari A, Zhou JY, Liew CW et al (2015) Compensatory islet response to insulin resistance revealed by quantitative proteomics. $\mathrm{J}$ Proteome Res 14(8):3111-3122. https://doi.org/10.1021/acs. jproteome.5b00587

19. Pearson GL, Mellett N, Chu KY, Boslem E, Meikle PJ, Biden TJ (2016) A comprehensive lipidomic screen of pancreatic beta-cells using mass spectroscopy defines novel features of glucosestimulated turnover of neutral lipids, sphingolipids and plasmalogens. Mol Metab 5(6):404-414. https://doi.org/10.1016/j. molmet.2016.04.003

20. Roomp K, Kristinsson H, Schvartz D et al (2017) Combined lipidomic and proteomic analysis of isolated human islets exposed to palmitate reveals time-dependent changes in insulin secretion and lipid metabolism. PLoS One 12(4):e0176391. https://doi.org/ 10.1371/journal.pone.0176391 
21. Caprioli RM, Farmer TB, Gile J (1997) Molecular imaging of biological samples: localization of peptides and proteins using MALDI-TOF MS. Anal Chem 69(23):4751-4760. https://doi.org/ 10.1021/ac970888i

22. McDonnell LA, Heeren RMA (2007) Imaging mass spectrometry. Mass Spectrom Rev 26(4):606-643. https://doi.org/10.1002/mas. 20124

23. Norris JL, Caprioli RM (2013) Imaging mass spectrometry: a new tool for pathology in a molecular age. Proteomics Clin Appl 7(1112):733-738. https://doi.org/10.1002/prca.201300055

24. Norris JL, Caprioli RM (2013) Analysis of tissue specimens by matrix-assisted laser desorption/ionization imaging mass spectrometry in biological and clinical research. Chem Rev 113(4):23092342. https://doi.org/10.1021/cr3004295

25. Wu CP, Dill AL, Eberlin LS, Cooks RG, Ifa DR (2013) Mass spectrometry imaging under ambient conditions. Mass Spectrom Rev 32(3):218-243. https://doi.org/10.1002/mas.21360

26. Spengler B (2015) Mass spectrometry imaging of biomolecular information. Anal Chem 87(1):64-82. https://doi.org/10.1021/ ac504543v

27. Minerva L, Clerens S, Baggerman G, Arckens L (2008) Direct profiling and identification of peptide expression differences in the pancreas of control and ob/ob mice by imaging mass spectrometry. Proteomics 8(18):3763-3774. https://doi.org/10.1002/pmic. 200800237

28. Djidja MC, Claude E, Snel MF et al (2009) MALDI-ion mobility separation-mass spectrometry imaging of glucose-regulated protein $78 \mathrm{kDa}(\mathrm{Grp} 78)$ in human formalin-fixed, paraffin-embedded pancreatic adenocarcinoma tissue sections. J Proteome Res 8(10): 4876-4884. https://doi.org/10.1021/pr900522m

29. Green-Mitchell SM, Cazares LH, Semmes OJ, Nadler JL, Nyalwidhe JO (2011) On-tissue identification of insulin: in situ reduction coupled with mass spectrometry imaging. Proteomics Clin Appl 5(7-8):448-453. https://doi.org/10.1002/prca.201000152

30. Minerva L, Boonen K, Menschaert G, Landuyt B, Baggerman G, Arckens L (2011) Linking mass spectrometric imaging and traditional peptidomics: a validation in the obese mouse model. Anal Chem 83(20):7682-7691. https://doi.org/10.1021/ac200888j

31. Grüner BM, Hahne H, Mazur PK et al (2012) MALDI imaging mass spectrometry for in situ proteomic analysis of preneoplastic lesions in pancreatic cancer. PLoS One 7(6). https://doi.org/10. 1371/journal.pone.0039424

32. Thiery-Lavenant G, Zavalin AI, Caprioli RM (2013) Targeted multiplex imaging mass spectrometry in transmission geometry for subcellular spatial resolution. J Am Soc Mass Spectrom 24(4): 609-614. https://doi.org/10.1007/s13361-012-0563-z

33. Janson ET, Comi TJ, Rubakhin SS, Sweedler JV (2016) Single cell peptide heterogeneity of rat islets of Langerhans. ACS Chem Biol 11(9):2588-2595. https://doi.org/10.1021/acschembio.6b00602

34. Djidja M-C, Claude E, Snel M et al (2010) Novel molecular tumour classification using MALDI-mass spectrometry imaging of tissue micro-array. Anal Bioanal Chem 397(2):587-601. https://doi.org/ 10.1007/s00216-010-3554-6

35. Eberlin LS, Margulis K, Planell-Mendez I et al (2016) Pancreatic cancer surgical resection margins: molecular assessment by mass spectrometry imaging. PLoS Med 13(8):e1002108. https://doi.org/ 10.1371/journal.pmed.1002108
36. Yin R, Kyle J, Burnum-Johnson K et al (2018) High spatial resolution imaging of mouse pancreatic islets using nanospray desorption electrospray ionization mass spectrometry. Anal Chem 90(11): 6548-6555. https://doi.org/10.1021/acs.analchem.8b00161

37. Brissova M, Haliyur R, Saunders D et al (2018) Alpha cell function and gene expression are compromised in type 1 diabetes. Cell Rep 22(10):2667-2676. https://doi.org/10.1016/j.celrep.2018.02.032

38. Hart NJ, Aramandla R, Poffenberger G et al (2018) Cystic fibrosis-related diabetes is caused by islet loss and inflammation. JCI Insight 3(8):e98240. https://doi.org/10.1172/jci. insight. 98240

39. Prentice BM, Caprioli RM (2016) The need for speed in matrixassisted laser desorption/ionization imaging mass spectrometry. J Postdoc Res 4:3-13

40. van Meer G, Voelker DR, Feigenson GW (2008) Membrane lipids: where they are and how they behave. Nat Rev Mol Cell Biol 9(2): 112-124. https://doi.org/10.1038/nrm2330

41. Maedler K, Oberholzer J, Bucher P, Spinas GA, Donath MY (2003) Monounsaturated fatty acids prevent the deleterious effects of palmitate and high glucose on human pancreatic beta-cell turnover and function. Diabetes 52(3):726-733. https://doi.org/10.2337/diabetes. 52.3 .726

42. Dotta F, Tiberti C, Previti M et al (1993) Rat pancreatic ganglioside expression: differences between a model of autoimmune islet $\mathrm{B}$ cell destruction and a normal strain. Clin Immunol Immunopathol 66(2):143-149. https://doi.org/10.1006/clin.1993.1018

43. Dotta F, Previti R, Neerman-Arbez M et al (1998) The GM2-1 ganglioside islet autoantigen in insulin-dependent diabetes mellitus is expressed in secretory granules and is not beta-cell specific. Endocrinology 139(1):316-319. https://doi.org/10.1210/endo.139. 1.5708

44. Saito M, Sugiyama K (2000) A distinct ganglioside composition of rat pancreatic islets. Arch Biochem Biophys 376(2):371-376. https://doi.org/10.1006/abbi.2000.1729

45. Dotta F, Colman PG, Lombardi D et al (1989) Ganglioside expression in human pancreatic islets. Diabetes 38(11):1478-1483. https://doi.org/10.2337/diab.38.11.1478

46. Saito M, Ito M, Sugiyama K (1999) A specific loss of c-series gangliosides in pancreas streptozotocin-induced diabetic rats. Life Sci 64(20):1803-1810. https://doi.org/10.1016/S0024-3205(99) 00122-8

47. Dotta F, Peterson LB, Previti M et al (1992) Pancreatic islet ganglioside expression in nonobese diabetic mice: comparison with C57BL/10 mice and changes after autoimmune beta-cell destruction. Endocrinology 130(1):37-42. https://doi.org/10.1210/endo. 130.1.1727711

48. Steiner DJ, Kim A, Miller K, Hara M (2010) Pancreatic islet plasticity interspecies comparison of islet architecture and composition. Islets 2(3):135-145. https://doi.org/10.4161/isl.2.3.11815

49. Dolenšek J, Rupnik MS, Stožer A (2015) Structural similarities and differences between the human and the mouse pancreas. Islets 7(1): e1024405. https://doi.org/10.1080/19382014.2015.1024405

Publisher's note Springer Nature remains neutral with regard to jurisdictional claims in published maps and institutional affiliations. 